\title{
Needs and availability of snake antivenoms: relevance and application of international guidelines
}

\section{Original Article}

\author{
Laura Scheske*, Joost Ruitenberg, Balram Bissumbhar
}

\begin{abstract}
Background: Snakebite has recently been declared a global public health emergency. Empirical data showing the true burden of snakebite is lacking. Treatment with specific antivenoms is considered the only cure. However, several factors have led to an ongoing antivenom crisis. This study offers recommendations concerning the improvement of antivenom access and control, by providing an overview of the factors limiting the successful implementation of international guidelines within the international industry and state institutions. It further investigates the reasons for the epidemiological knowledge gap regarding snakebites.

Methods: Data for this study was collected using surveys with closed- and open-ended questions, which allowed for descriptive and thematic analysis, respectively. Participants for this study were selected as follows: 46 manufacturers were contacted from the open-access World Health Organization (WHO) Database for antivenom producers; 23 National Health Authorities (NHAs) of high-burden countries were contacted; and 11 poison centers or experts were randomly contacted. Results: In total, responses from 6/46 (13\%) manufacturers, 10/23 (43\%) NHAs, and 3/11 (27\%) poison centers were received. The low response rates had a limiting effect on the coverage of this study, allowing only exploratory conclusions to be drawn. Based on the gathered information, a probable reason for the epidemiological knowledge gap is the low priority given to snakebites on public health agendas, driving interest and funding away from research in this field. As a consequence, the ensuing lack in funding is preventing state institutions and manufacturers from implementing international guidelines to the highest standards. Furthermore, manufacturers indicated that international guidelines were often not applicable in the field, lacking technical information and protocols.

Conclusion: Snakebite ranks low on international public health agendas, and partially due to this low priority, NHAs have shown limited efforts in conducting epidemiological studies, training health workers on snakebite management and creating national snakebite management strategies. The lack of NHA involvement is reflected in poor access to appropriate antivenoms as well as a lack of antivenom regulation. Manufacturers are taking positive steps toward full implementation of international guidelines and are improving quality control procedures. However, in order for international guidelines to become truly useful in the field, more technical guidance is required. This study reflects that there is a general lack of knowledge transfer amongst various actors: most producers, health authorities, and experts expect increased and improved communication and guidance from leading international bodies. Due to the low response rates observed in this study, conclusions drawn herein are not representative of the global situation; yet provide an exploratory insight on the difficulties facing antivenom management.

Keywords: Snakebite, Antivenom, Envenomation, Guidelines, Neglected Tropical Disease (NTD), International Public Health Copyright: @ 2015 by Kerman University of Medical Sciences

Citation: Scheske L, Ruitenberg J, Bissumbhar B. Needs and availability of snake antivenoms: relevance and application of international guidelines. Int J Health Policy Manag. 2015;4(7):447-457. doi:10.15171/ijhpm.2015.75
\end{abstract}

\section{Article History:}

Received: 20 December 2014 Accepted: 30 March 2015 ePublished: 4 April 2015

\section{*Correspondence to: \\ Laura Scheske}

Email: lscheske@gmail.com

\section{Key Messages}

Implications for policy makers

- Inconsistent record keeping and incomplete hospital registers play a big role in the lack of epidemiological knowledge about snakebite. In addition to improving case reporting systems by health services, the burden of snakebite may be assessed for specific foci using community-based epidemiological studies. Patient records and death certifications must be improved (e.g. to use International Classifications of Diseases code T63.0 to certify death caused by snake envenoming).

- Teaching health workers the rational use and administration of antivenoms and proper snakebite identification will save lives and reduce antivenom waste.

- More research must be supported. Including: developing treatments to prevent local tissue damage, clinical trials for existing antivenom products, and perhaps even a conceptual change in fundamental antivenom design.

- International networks for knowledge transfer must be established, or existing networks strengthened: improve communication between existing research groups by World Health Organization (WHO) coordination, hold a follow up conference on the WHO Guidelines.

- The addition of snake antivenoms to the WHO Prequalified Medicinal Products list could help ensure good quality, safe, and effective antivenoms for global use.

- Stricter international regulation of the antivenom market would prevent the export of non-efficacious products between countries and improve the quality of antivenom products.

Implications for public

This study provides an overview of the current global situation of snake antivenom management. Venomous snakebites kill an estimated 100,000 people per year. Antivenoms are considered to be the only specific cure for snakebite envenomation. The technology for their production exists, yet appropriate distribution mechanisms and international knowledge transfer are lacking. This study appeals to the international public health community to renew efforts for a global antivenom strategy, to provide access to safe and effective antivenoms for the populations in need. 


\section{Background}

Snakebite envenomings kill one tenth as many people as malaria - an estimated 100,000 a year $(1,2)$. Recently added to the World Health Organization (WHO) list of Neglected Tropical Diseases (NTDs), snakebite primarily affects poor rural populations in developing countries, who have limited access to appropriate antivenom treatment. The situation of snakebites, snakebite management and antivenom supply has been declared a global public health emergency (3).

Snakebite is a recognized medical emergency in those parts of the world inhabited by venomous snakes. It generally affects people living in rural communities, with children and agricultural workers most at risk $(3,4)$. Estimates show that snakebites occur most in South and Southeast Asia, in SubSaharan Africa, and Latin America $(2,3,5)$. According to existing studies, India suffers most from snakebite, with as many as 50,000 snakebites a year, resulting in up to 11,000 deaths (5).

Incidence of snakebite fluctuates with seasonal changes, as climate changes influence annual cycles of agricultural activity $(6,7)$. In Nigeria, for instance, as many as $70 \%$ of hospital beds can be occupied by victims of snakebite during the sowing and harvesting seasons (8). Furthermore, the impact of envenomings is much higher than case numbers suggest: an estimated 2 million Disability-Adjusted Life Years (DALYs) are lost per year for Sub-Saharan Africa alone, since the majority of victims are young, and many are left with permanent sequelae or incapacitating consequences of envenoming (3). This in turn can have a significant impact on local economies, as entire communities are dependent on the products of agricultural workers (3).

The true burden of snakebite has not been accurately measured using empirical studies, resulting in a substantial epidemiological knowledge gap in this field $(2,9,10)$. This lack of empirical data has led to the negligence of snakebite on global health agendas, and a lack of interest in snakebite research, antivenom development, and financing.

Snake antivenom immunoglobulins, obtained by immunizing horses with selective snake venoms, are considered the only specific treatment for snakebite envenoming (2). The WHO has estimated that 10 million vials of antivenoms are needed each year to control the effects of snakebite envenomings (3). The 46 laboratories dedicated to producing antivenoms are unable to meet this demand $(3,11)$. Due to the neglected status of snakebites, governments and policy-makers largely ignore antivenoms, keeping them low on public health agendas and far away from national budgets $(3,11)$. To improve global availability, larger laboratories require clearer descriptions of market sizes and needs, in order to plan longterm manufacturing strategies $(3,10)$.

The end of the 20th century saw a dramatic reduction in antivenom producers: Behringerwerke AG in Germany, which produced antivenoms for Africa and the Middle East, stopped production in the 1980s, South African producers have been fraught with difficulties, and CSL Australia stopped research activities into antivenoms, yet continues production only for Australian antivenoms (12). Production expenses, production complexity, and lack of a lucrative market largely drove such shutdowns. Integrating antivenom production into a global strategy to combat snakebites may be the key in preventing further reduction of producers in the 21 st century $(11,13)$.

The aforementioned drawbacks point to a central problem faced by antivenoms: access. Low production, inadequate distribution chains, and the need for species and regionspecific products thwart global antivenom availability (14-16). The result of low output and high production costs are antivenoms that are unaffordable to those who need them most. There is a global deficiency of appropriate antivenom supply, and the poor rural areas most burdened by snakebite, such as Sub-Saharan Africa and parts of Asia suffer most from this limited availability, resulting in poor treatment outcomes $(8,12,17)$.

A commendable step was taken with the publication of the WHO Guidelines on production, control and regulation of snake antivenom immunoglobulins in 2008 (18). However, possibly due to the absence of follow up meetings or updates since, problems in the implementation of these guidelines in high-burden regions have arisen. In order to combat some issues of access, the WHO has included snake antivenom immunoglobulins in the WHO Model List for Essential Medicines, encouraging countries to do the same and thereby ensuring national antivenom stocks; this has yet to show an increase in global access to antivenoms $(11,12,14)$. The reasons behind this have not yet been thoroughly researched, creating a need to investigate the barriers to antivenom accessibility. This study focuses on the extent to which the international industry and state institutions have been able to implement and put to use the WHO Guidelines. Further emphasis is placed on the factors behind the epidemiological knowledge gap regarding snakebites, and what countries are doing to improve data collection. The study also identifies factors impeding global antivenom accessibility, and analyzes how manufacturers cope with stringent quality control measures, and increasing demand. Recommendations based on the research findings are provided at the end.

\section{Objective}

The objective of the present study is to make recommendations concerning the improvement of antivenom access and control globally, by providing a clear overview of the factors limiting the successful implementation of WHO Guidelines within the international industry and state institutions. A second objective is to investigate the factors causing the epidemiological knowledge gap regarding snakebites.

\section{Methods \\ Selection of participants}

Study participants were selected for three stakeholder categories: manufacturers, National Health Authorities (NHAs), and poison centers or experts. Participants included representatives from all WHO regions. All known $(\mathrm{N}=46)$ antivenom manufacturers were contacted based on the open-access WHO database of antivenom producers (19). NHAs from 23 high-burden countries were contacted, based on the attendance list of the WHO Bi-Regional Workshop on Production, Control, and Regulation of Antivenoms (2008, Jakarta), and 11 poison centers or experts from highburden regions were contacted. See Appendix 1 for a list of manufacturers contacted, and Appendix 2 for a list of 
countries in which NHAs were contacted.

\section{Surveys}

The surveys contained open- and closed-ended questions, and were designed to gather a broad range of information: included were questions on epidemiology, access to antivenoms, antivenom production, as well as WHO Guideline implementation. Each survey was designed to gather as much information as possible from the relevant actors (NHAs, manufacturers, and poison centers or experts), so as to acquire a complete overview of the situation regarding the knowledge surrounding snakebites as well as strategies for the production or distribution of antivenoms. For member states that were not implementing the WHO Guidelines, the aim was to establish the impeding factors as well as the type of technical support required for successful implementation. The surveys also required participants to list all venomous snake species, as well as all antivenoms produced in their countries to the best of their knowledge. This information was later crosschecked with WHO databases.

To increase survey response rate, each survey was accompanied by an explanatory cover letter. The questionnaires were made available both in French and English so as to increase response from francophone countries. Reminders were sent out to all survey recipients twice by email, and, following continued non-response, participants were called personally.

Full surveys used for this study can be found in Additional files $1-3$.

\section{Data analysis}

Data analysis of the surveys was conducted using the EpiInfo statistical program, developed by the Center of Disease Control (CDC). This study mainly comprised exploratory and descriptive research, in which descriptive statistics are used for analysis of single variables. The closed questions required primarily bivariate responses, i.e. yes or no, and rating on a scale in one instance. The bulk of data collected in the surveys came from open questions in which participants were requested to either answer in lists, or write short responses. These answers were analyzed on an individual basis, checked for relevance to the question, as well as use of English. Since most of the open questions were intricately connected in meaning and context, all open answers were analyzed with an eye to the responses of the entire survey.
All data was exported to Microsoft Excel for tabulation, double-checking and control.

Results

Study participation

Study participation from the three different actor groups: NHAs, manufacturers, and poison centers, varied considerably. Figure 1 provides an overview of the response rates for the different stakeholder groups.

NHAs from 23 countries were approached for participation in the study. The response rate came to $43.5 \%$, meaning that responses from 10 countries were received.

The 46 global manufacturers of antivenom were contacted using the contact information provided in the WHO online database (19). Unfortunately, 23 emails did not reach the intended participants, as their contact details were invalid, out of date, or simply not listed. This in itself is a sign that no efforts have been put into maintaining an up-to-date database. Out of the remaining 23 manufacturers that presumably did receive our emails, only seven sent back responses, leading to a response rate of $30 \%$ for manufacturers. The absolute response rate was $26 \%$, however, because two individuals from the same institute provided responses; these responses were combined and regarded as a single entity. It is important to note that the manufacturer responses in this study only represent $13 \%$ of the total number of global antivenom producers. Therefore, conclusions based on these responses should only be made tentatively.

Poison centers or experts from 11 countries were approached to obtain supplementary information on snakebite and antivenom production in their region. Three surveys were sent back, resulting in a $27 \%$ response rate for this group. However, the majority of NHAs, manufacturers, poison centers and even experts on the topic did not respond upon repeated contact over an extended period of time.

Appendix 1 and 2 provide a list of the NHAs and manufacturers that were contacted for this study and denotes study participants.

\section{Lack of epidemiological knowledge}

NHAs were asked to rank the priority of snakebites in their national health agendas on a scale from $1-5$. Where 1 is given lowest priority and 5 is the highest priority. Without exception, all NHAs ranked snakebites as having low priority, with an average grade of $1.38 / 5$. The fact that such low

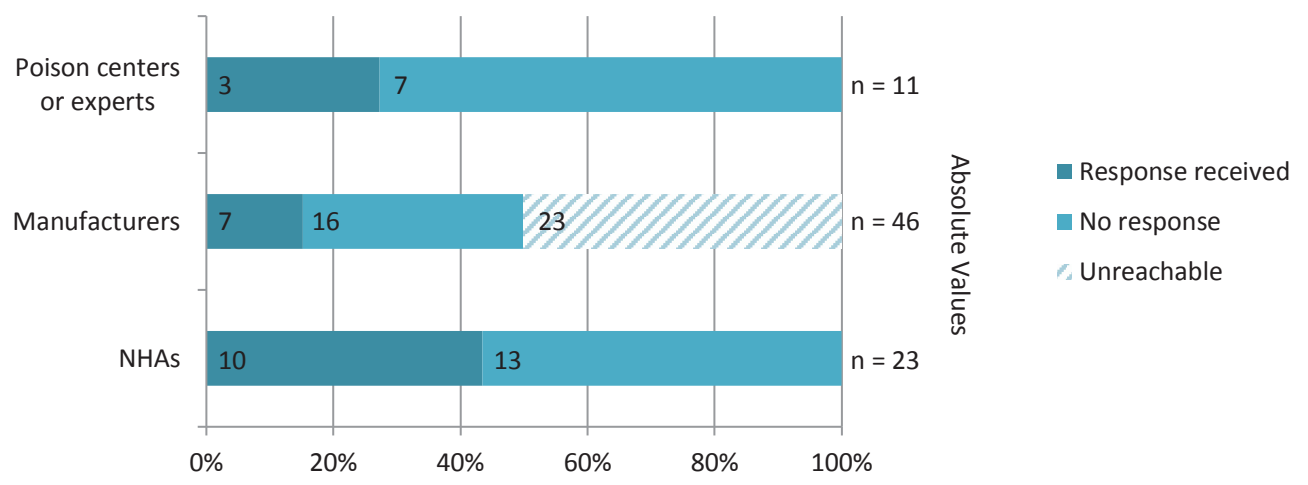

Figure 1. Study response rates organized by actor groups: NHAs, manufacturers and poison centers or experts. 
priority is given to snakebites in the 10 countries that partook in this study, may point to an underlying reason for a global lack of epidemiological knowledge on the topic.

Furthermore, only three countries (30\%) were able to provide indications of snakebite incidence and mortality in their national setting. The cumulative incidence for these three countries was 38,343 bites per year. These results strongly support the current notion that global cases of snakebites are underestimated on a grand scale.

Six $(60 \%)$ of the NHAs indicated that health workers in their countries do not receive special training on topics regarding snakebite. The remaining $40 \%$ that do train their health workers indicated that mainly doctors and pharmacists receive special training on venomous animal bites, and that only some first aid courses cover treatment of envenomed patients.

The majority (70\%) of the NHAs that we surveyed indicated that their countries require certification for the cause of death of a patient. For the remaining 30\% of NHAs, this information was missing. Although the cause of death needs to be certified, it is likely that most snakebites go by unnoticed, as health workers are not sufficiently trained in the identification of envenomed patients. Furthermore, even if snakebites are correctly identified, only four of the countries we surveyed indicated that snakebite cases must be reported to the authorities. In addition, only two countries mentioned having a central register in which all cases of snakebite are recorded.

\section{Approaches used to improve epidemiology}

NHAs were asked whether their countries have ever performed any epidemiological surveys on snakebites in the past, and whether there are currently any efforts to improve epidemiological knowledge of snakebites. Of the ten NHAs that participated in our study, only three (30\%) indicated that epidemiological surveys on snakebites had been performed in their countries. Four countries (40\%) had never conducted epidemiological surveys, and for the remaining three (30\%) data were missing.

The existence of current efforts to improve epidemiological knowledge was also questioned, and four countries (40\%) indicated that efforts were indeed taking place.

\section{Implementation of World Health Organization (WHO) Guidelines}

One of the primary objectives of this study was to find out whether, and to which extent, the WHO Guidelines for antivenoms were being implemented in selected countries. Both, NHAs and manufacturers were questioned about their knowledge concerning the guidelines.

Pooled into one group, responses from NHAs and manufacturers from 15 different countries were obtained. In total, $86.7 \%(n=13)$ cited being aware of the WHO Guidelines, and only two countries (13.3\%) were not aware of the guidelines, see Figure 2. More importantly, eight of the 15 countries that participated had never implemented WHO recommendations, and seven said they had. Below, we look in detail at the results found on WHO Guidelines, and differentiate between NHA and manufacturer responses:

\section{National Health Authorities (NHAs)}

Of the ten NHAs that responded to our survey, only two (20\%) said they were unaware of the WHO Guidelines, and the remaining $80 \%$ indicated awareness. Interestingly, five (50\%) responders indicated that they have national expert groups responsible for work surrounding the guidelines. However, the composition of these expert groups remains unclear, and it is possible that some responders misunderstood the question and in their answers referred to a general health department, or an individual snake venom expert known to them.

\section{Manufacturers}

All six (100\%) manufacturers who responded to the survey said they were aware of the WHO Guidelines. This may indicate that producers of antivenoms, due to their specialization, are more fluent in staying up to date with developments in their field, while NHAs might neglect topics that are not a priority on their agendas. Nonetheless, we cannot draw adequate conclusions from these responses, as these six manufacturers represent only $14 \%$ of the global total.

\section{Points of implementation \\ National Health Authorities (NHAs)}

Eighty percent $(n=8)$ of NHAs indicated that they were aware of the WHO Guidelines. Of these, 37.5\% $(n=3)$ said that they had implemented some recommendations from the guidelines, and the remaining $62.5 \%(n=5)$ said they had not. As expected, the two NHAs who were unaware of the WHO Guidelines correspondingly also did not have an expert group, nor did they indicate guideline implementation.

The specific recommendations that have been implemented by countries include antivenom registration, production guidelines and quality control of antivenoms. Respondents

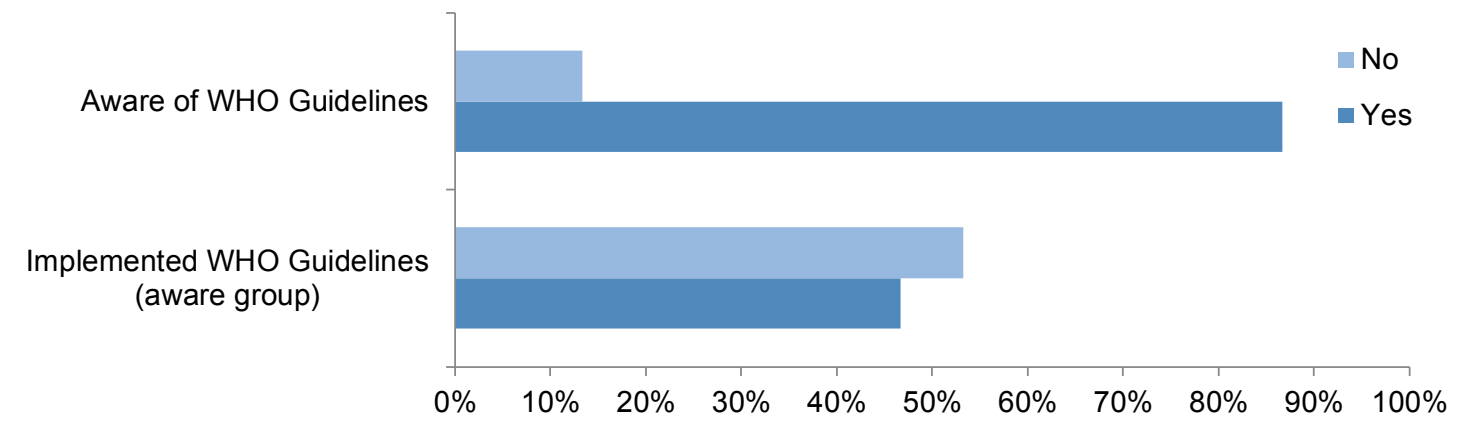

Figure 2. Awareness of WHO Guidelines among NHAs and manufacturers combined. 
noted that they use all sections of the guidelines to evaluate antivenom products prior to market authorization in their countries. Countries in which no recommendations had been implemented cited problems in their national health infrastructure, lack of technical knowledge to initiate own production of antivenoms, and irrelevance of guidelines according to policy-makers. Since the 10 NHAs that participated in this study represent only a fraction of the total number of high-burden snakebite countries, it remains unclear to which extent NHAs have been able to implement WHO Guidelines in their national settings.

\section{Manufacturers}

Five manufacturers (83\%) indicated that they had implemented some of the WHO recommendations at their facility. Only one manufacturer cited no implementation of the recommendations. However, the manufacturer explained that they follow local Good Manufacturing Practice (GMP) and pharmacopoeia guidelines, which are often adapted using WHO GMP guidelines for biologicals i.e. the WHO Guidelines on snake antivenoms have not been specifically implemented, but the WHO machinery is wellestablished and acknowledged by regulatory officers. These five manufacturers represent $10 \%$ of the total number of antivenom manufacturers, and it is unclear how successful other manufactures have been in adopting the WHO Guidelines.

Since the WHO Guidelines have been written from an inclusive perspective, covering many antivenom production methods, various facilities were already applying some of the recommendations. Most manufacturers explained that they refer to the WHO Guidelines for the construction of GMP production facilities, as well as streamlining quality control procedures and testing the final product. Further recommendations that different facilities implemented include proper documentation of final products, characterization of starting venom batches, horse immunization procedures, plasma pooling, as well as various in-process analyses, and sample retention schemes. Most manufacturers also cited being unable to comply with some of the WHO recommendations, as their facilities lack certain technical equipment, or funds to upgrade to the latest machines. One aspect that remains least implemented is the viral-validation process, as this requires highly specialized equipment.

\section{Technical help required}

Seven $(70 \%)$ of the NHAs indicated that they would require external assistance for the implementation of all or some of the recommendations presented in the WHO Guidelines. Only two countries, both with very well-established antivenom production and management systems, indicated that they would not require external assistance for the implementation of the guidelines. For one country data was missing, however this specific respondent was only able to fill in the second half of the survey, as they were uninformed about all sections of the survey except for Access to Antivenoms. It is highly likely that this country too, is in need of external assistance to implement the guidelines. Four of the NHAs that cited requiring external assistance to implement WHO recommendations also provided details about the type of support they required: the most common request was for technical support to adapt WHO Guidelines to national settings, as well as help and funding in conducting training programs for regulatory officers, health workers, and the public, on topics surrounding snakebites. In addition, respondents requested support to set up or streamline production facilities, and support in antivenom quality control including the safety and efficacy of unevaluated imports.

Further aspects in need of external assistance included: improved access to information, know-how, networking, funding for epidemiological and clinical studies, technical support with regards to information material (leaflets, posters, brochures), creating awareness for prioritizing antivenom use.

Interestingly, poison center/expert respondents revealed that they would like some kind of acknowledgement for groups who are providing efforts in the management of snakebites and antivenoms. This ties in closely with the requests for improved networking and knowledge transfer. Furthermore, respondents said that they would like direct instructions from WHO offices to help them initiate appropriate, and country specific solutions.

Compared to the NHAs, fewer antivenom manufacturers cited the need for external assistance to implement the WHO Guidelines. Nonetheless, half of the manufacturers did indicate the need for external guidance. Most commonly mentioned aspects involved the provision of specific technical protocols that can be followed in the field. These included training of staff for caprylic acid precipitation and plasmapheresis, consultants for viral reduction processes and for acquiring appropriate GMP approved equipment, consultants for capacity building surrounding laboratory animals including the use of non-equine animals, and consultants for the production of polyvalent antivenoms. In general, all manufacturers cited the need for support in acquiring the latest technology on antivenom production, the best production equipment, as well as in constructing appropriate production facilities. One manufacturer also indicated that they require guidance for appropriate, clear, and cost-effective package design.

\section{Quality control of antivenoms}

One of the main challenges within the global antivenom market is the production and trade of suboptimal antivenom preparations $(7,12,20)$. Snake antivenoms are currently not prequalified by the $\mathrm{WHO}$, allowing manufacturers to dispatch products as they see fit. Due to inter- and intraspecies specificities in venom composition, a key aspect in antivenom quality control lies in the evaluation of the neutralizing capacity of antivenoms for the venoms of snakes in a specific country or region $(7,18,20)$. The WHO guidelines provide detailed methodologies of preclinical efficacy tests that are necessary to ensure the neutralizing efficacy of antivenoms for specific snake species, however it is unknown whether manufacturers have been able to follow all these recommendations $(7,18,20)$. The lack of national and international regulation of antivenoms results in suboptimal products being made available on the market (11). A negative consequence is that countries without local manufacture of 
antivenoms purchase products, which do not match their local snake population. Those that end up being affected by this global mismanagement are the patients, whose mistreatment may trigger distrust in the health systems.

\section{Quality control at production facilities}

All of the manufacturers surveyed indicated having some sort of quality control procedure in their production process. All of the manufacturers also indicated having an external quality control department responsible for testing. The manufacturers stated that they follow national or international pharmacopoeia guidelines for good laboratory practices and GMP. Finally, all the final antivenom products produced by the facilities surveyed undergo rigorous testing (including Pyrogen test, potency test, abnormal toxicity, sterility, $\mathrm{pH}$, appearance).

In general, it appears that the manufacturers that partook in this study are well-equipped for quality control testing. In support of this claim, we also found that all manufacturers were subject to quality control measures set by national regulatory guidelines. Most manufacturers mentioned stringent GMP guidelines, facility requirements, environmental regulations, as well as packaging regulations. The manufacturers also indicated having to send batch documentation and regular quality control reports to NHAs. All but one manufacturer said they have to undergo regular inspections by NHAs, during which all GMP and quality system aspects, for the entire production chain are tested. The one manufacturer that indicated not undergoing regular inspection noted that they were pending application submissions to their FDA, and that inspections would commence in 2014.

In accordance with the results received from manufacturers, our NHA respondents indicated that those countries with local manufacture of antivenoms do possess national regulatory guidelines, and inspect manufacturing facilities on a regular basis. Unsurprisingly, countries without local manufacture of antivenoms indicated not having any regulatory guidelines for the production of antivenoms.

\section{Use of reference preparations}

The creation of reference preparations for snake venoms or antivenoms is a topic that is widely discussed in the current literature, and has been recommended by the WHO on several occasions $(12,18)$. Of the manufacturers that partook in this study, all but one indicated that they only used in-house reference preparations of venoms and antivenoms to ensure consistency of their products. One of these manufacturers indicated that they were in the process of nationalizing their in-house reference standards so that these could be used nationwide. The last manufacturer said that they do not use any reference preparations in their production process. Since the majority of manufacturers only test their antivenom batches against in-house reference preparations, it is difficult to compare antivenoms on a national, or even international level, and for buyers to get an idea of what an ideal product should consist of. Nonetheless, the use of in-house reference preparations may be a first step towards generation of national reference preparations (20).

In accordance with our results from the manufacturer survey,
90\% $(n=9)$ of the NHAs surveyed said that they had no national reference preparations of snake venom or antivenoms available, with the exception of one. These numbers come as no surprise, since the majority of responses came from countries that do not produce antivenoms locally. Yet, of the three responding countries that do produce antivenoms, only one had national reference preparations.

These results bring to light the underlying problems of lack of funding and low priority given to snakebite and antivenom research across the globe.

\section{Access to antivenoms}

A key issue that remains in the treatment of snakebites is limited access to antivenoms $(8,16)$. It is generally agreed upon that quick treatment of an envenomed patient with appropriate antivenom substantially increases rates of survival and reduces morbidity $(14,21)$. Most countries with a high burden of snakebite do not have the infrastructure or market size to support local antivenom production (3). Furthermore, many important manufacturers have shut down or been privatized. As a consequence many countries still struggle with providing effective, appropriate, and affordable antivenoms for their citizens.

\section{Snake antivenoms as Essential Medicines}

All of the countries from which we received NHA responses operated with an essential medicines list. Snake antivenoms were found on $70 \%(n=7)$ of the national essential medicines lists. Two respondents cited not listing antivenoms as essential medicines, and one respondent did not have this data available. The fact that snake antivenoms are already found on the majority of essential medicines lists in countries that participated in this study, representing different WHO regions, is an optimistic step towards improving access to antivenoms. However, conclusions should only be cautiously drawn, as these seven countries represent a small fraction of those with a high burden for snakebite.

\section{Antivenoms for different snake species}

Antivenoms are not universally effective, creating a need to produce antivenoms for different snake immunotypes, which are inter- and intra-species specific $(7,20)$.

Only $33 \%(n=2)$ of the manufacturer respondents indicated that they produce antivenoms for all medically important snakes in their countries. The remaining 66\% $(n=4)$ indicated that they were only able to produce antivenoms for the medically most important snakes. Manufacturers cited reasons such as scarcity of venom samples, difficulties to breed snakes, as well as lack of funds for not being able to produce antivenoms for all snake species.

All manufacturers were aware of the intra-species venom differences from snakes inhabiting different regions. Fifty percent of the manufacturers said that they produce antivenoms that take into account these differences in immunotypes. However, only one manufacturer offered an explanation of how they ensure that there is sufficient crossreactivity of their antivenom products for the different venom immunotypes: they sourced venom animals from across the species range to cover the geographical variation. At facilities where snakes are farmed and bred on site, it is possible that 
effectiveness of the resulting antivenoms decreases for the same snake species in the wild, meaning that the potency of the antivenoms in real situations may become suboptimal.

\section{Production output and prices}

There exists a wide range of antivenom producers across different regions: from facilities in India producing over one millionvialsayear, to small-scale, privateproducersin Australia, producing $<5,000$ vials a year. Most of these manufacturers focus on producing antivenoms for their geographical regions, however some also export internationally. The five manufactures that provided production data represent $11 \%$ of the total number of producers worldwide, and their output amounts to approximately $15 \%$ of the total need for antivenoms. If the remaining 40 antivenom producers manage to produce similar total amounts, it should be possible to achieve the global target of $10,000,000$ antivenom vials per year.

Moreover, antivenom prices vary across regions. The cost for one vial of antivenom ranged from $€ 7.00$ per vial from a producer in Pakistan, to $€ 25$ per vial from a producer in Thailand, to $€ 1500.00$ per vial from an Australian producer. The therapeutic price for a treatment using antivenoms can be up to ten times higher than the price for a single vial, as the severity of envenoming affects the amount of antivenom required. Moreover, export prices are usually 50\%-200\% higher than local prices. The huge price discrepancy between different producers is most likely due to the fact that those producers selling at low prices are heavily government subsidized or even government run, whereas expensive antivenoms are produced by privatized facilities. Although some of the prices for antivenoms appear low from a western perspective, $€ 7$ is a huge price to pay for persons living in poverty, or at about $€ 1$ a day.

\section{Coping with demand}

Four $(66 \%)$ of the manufacturers that responded to our survey said that their production outputs depend on clinical needs of antivenoms in their countries. One manufacturer indicated that their production did not depend on national clinical needs, and one did not provide this data. Three (50\%) of the manufacturers said that they were able to cope with the demand placed on them by their NHAs, two said they were unable to cope, and for one data was missing. The two manufacturers that were unable to cope with demand explained that they were the only producers in their countries, and that there were no other producers, even in the private sector. Fortunately, both cited that they were either undergoing renovations at their current facilities, or building new laboratories and stables to expand their output.

Three $(50 \%)$ of the manufacturers that responded to our surveys are large-scale producers that export antivenoms worldwide. The other half indicated that their facilities only qualified as small-scale manufacturer, and did not have the means, or output for international export. The three manufacturers eligible for international export indicated that they primarily exported to neighboring countries with the same snake species, or to zoos and institutions at which snakes from their countries are kept. One of the big manufacturers, located in India, listed several African countries as export destinations, yet they did not explain which antivenoms were exported, nor did they justify cross-reactivity.

\section{Discussion and Conclusion}

It has been one hundred and fifteen years since Calmette successfully treated a patient with anti cobra venom (22). Since then, antivenom technology has advanced little, and a lack of interest, investments, as well as low prospects of commercial returns, has resulted in a global antivenom crisis (12). The fact that more than 100,000 people are left to die due to snakebite envenomings reflects a global loss of momentum in snakebite research, antivenom development, and financing. Just forty-six antivenom producers are struggling to meet the demand of 10 million antivenom vials per year, unsuccessfully. There is a fundamental flaw in the global management of snakebite and antivenoms, and this study tries to offer a sliver of explanation as to what is going wrong.

The essence of this study was to provide general information about where snakebite and antivenoms rank on public health agendas, how much is known about these topics, whether access to efficacious antivenoms may become a reality, and whether the international industry and state institutions have been able to implement international guidelines.

The main difficulty that was encountered during the study was that many intended participants were unreachable, due to expired contact details provided in the WHO webdatabase. When trying to contact the global manufacturers of antivenoms, half of the emails did not go through, and from the remaining half that did go through; recipients were very reluctant to respond. This study extended over a prolonged period of time, during which intended participants were repeatedly contacted by different means, and encouraged to send their responses. It is a fact that the majority of recipients, already involved in snakebite or antivenom research, refused to participate. If this non-response is due to lack of interest, the continuation of this apathetic behavior will only exacerbate problems in the long run. Due to the low response rates encountered in this study, the coverage of the results presented here is not universally applicable to the global situation of antivenom management. Nonetheless, the results provide focused empirical data that verify what others have discussed before; the lack of momentum and resources made available to combat this neglected disease $(12,13,17)$.

In the course of this research, it quicklybecame clear that there is an absence of empirical data of the global snakebite burden. The results indicate that a probable reason for the epidemiological knowledge gap is the low priority given to snakebites on public health agendas. Unfortunately, high priority is unlikely to be given to a disease lacking epidemiological data, and epidemiological studies for low priority issues are unlikely to be funded. Furthermore, the majority of countries that partook in this study do not train their health workers specifically about snakebite management, nor do they require snakebites to be reported to authorities. The surveys showed that snakebite management in most participating countries is patchy at best. An applauded achievement lies in the recent addition of snakebite to the WHO list of NTDs, which may garner increased attention and perhaps even funding from the international community $(7,13)$.

The findings in this study verify the notion of a global 
antivenom crisis; they are still largely inaccessible to those who need them most, and often traded inefficiently (8). Even when available, selling prices of a single vial of antivenom far exceed the income of those living at or near the poverty line, and that is disregarding the fact that several vials are required for successful treatment. The results clearly indicate that privatization, and small production output drive up prices to unrealistic amounts, and it appears that only heavy subsidies can outweigh these costs.

The respondents to our manufacturer surveys were very limited. It is highly likely that the results obtained from such a low response rate are not applicable to all manufacturers, and should not be applied to the global situation. It is largely understood that the 46 manufacturers of antivenoms worldwide are not able to cope with global demand (12). Wellestablished institutions are making serious efforts to improve antivenom supply in their regions, but are unable to tackle the global crisis without increased international collaboration. Nevertheless, the manufacturers that partook in this study were located in countries with a range of economical backdrops, allowing for certain conclusions to be drawn. The findings point out that unequal resource allocation may be causing the inequality of antivenom supply i.e. that producers focus their production on antivenoms for snake species found in their vicinity, and may not have the resources available to produce antivenoms against snakes from other regions. This unequal resource allocation seems to be one of the main driving forces hindering the access to appropriate antivenoms. The region suffering most from this resource inequality is Africa, which continues to receive inappropriate antivenoms from foreign manufacturers (17).

A major problem that may result from the continued distribution of suboptimal antivenoms is that patients may lose trust in antivenom treatment methods. Simply put, if snakebite victims keep getting treated with inappropriate antivenoms and as a result die or suffer permanent sequelae, their families and friends may not believe that antivenoms are indeed effective. As a result, this could lead to a reversal of advancements seen in public health, and could even spill over to other treatment methods. It is likely that patients may want to seek out traditional healers instead (2). With an eye to this problem, several survey respondents suggested that antivenoms should be added to the WHO Prequalification of Medicinal Products Program, which would ensure that NHAs could purchase safe, effective and quality controlled products for their citizens.

A positive step towards quality global antivenom production was made with the publication of the WHO Guidelines (18). A major part of this research was to determine whether the guidelines have been implemented in the countries surveyed. What we found was that all manufacturers, we contacted were well aware of the WHO Guidelines, and that the facilities had already been using many of the recommended methods. More importantly, some of the facilities that partook in this study were contributors to the WHO Guidelines. All manufacturers indicated that they referred to the recommendations for the construction of GMP laboratories, and for streamlining quality control testing procedures on the final product. Nonetheless, not all manufacturers had been able to implement all of the recommendations, primarily due to a lack of exact manufacturing protocols or facility blueprints. Furthermore, most manufacturers cited financial restraints and lack of technical personnel to interpret and implement guidelines in national settings. Importantly, the existence of in-house quality control procedures or adherence to GMP guidelines, does not guarantee the production of efficacious antivenom products. Antivenom production requires vigorous preclinical testing that surpasses standard laboratory quality control measures, and takes into account the paraspecific neutralization levels for species-specific venoms (7).

An important consideration made in the WHO Guidelines concerned the establishment of international reference preparations for venoms and antivenoms. Our results show that only one participating country is on the path to creating national reference preparations, whereas the majority let producers use in-house reference standards. It will not be easy to create international reference standards for antivenoms due to the immunological differences in venom composition, however if countries manage to create national reference pools for each medically important snake species, that could eventually lead to an international stock of global reference standards, which can be used for further tests and assessments (18).

The NHAs reflected less technical knowledge about the WHO Guidelines, despite the presence of specific recommendations for national regulatory authorities such as those regarding distribution, management and control. Although $80 \%$ of the NHA respondents said they were aware of the guidelines, it is unclear to which extent recommendations have been implemented on governmental levels, as descriptions were scarce. Furthermore, $80 \%$ in this study only represents 8 countries in the world, which is a fraction of those burdened by snakebite worldwide. It appears that the WHO Guidelines have primarily been adopted in the antivenom production community, and remain largely disregarded on official levels. It appears that NHAs deem the WHO Guidelines to be irrelevant in their work, as the title attracts a more technical crowd, and may deter policy-makers. One issue that was mentioned repeatedly outside of the scope of the survey questions was the lack of knowledge transfer amongst institutions involved in snakebite or antivenom research and production. From a public health perspective, it is only beneficial for producers to mediate their own discussions and to actively provide technical assistance to less experienced producers. Lack of knowledge seems to be a recurring theme in the study of snakebites and antivenoms; on the one side we have policies obstructing progress, and on the other we have a free market economy that is creating unreasonable competition.

Almost seven years have gone by since the WHO Guidelines were published, and the document has not been updated. How is it that snakebite mortality accounts for one tenth as much mortality as malaria, but that there exists no global snakebite strategy that is comparable to global malaria strategies? Similarly, a global fund could be started for the procurement of antivenoms, which are then dispatched globally. Most of the current antivenom producers are also producers of vaccines, and there is no doubt that contacts to funders and distributers exist. With this backdrop, it only seems appropriate for the WHO to initiate a coordinated approach that will connect the 
scattered dots.

Snakebite is treatable. The means to reduce morbidity and mortality exist. A much stronger international initiative is needed to focus the attention of international organizations, government agencies, research institutes, and funders on snakebites and antivenoms.

\section{Ethical Issues}

The study protocol was reviewed by the Athena Institute for Innovative and Transdisciplinary Research in Health Sciences, VU University, Amsterdam, The Netherlands, and no ethical concerns were raised. The authors received no specific funding for this study.

\section{Competing interests}

Authors declare that they have no competing interests.

\section{Authors' contributions}

Conceived and designed the study: LS, JR, BB. Performed data collection and analysis: LS. Wrote the paper: LS. Review of paper: JR, BB, LS.

\section{Additional files}

Additional file 1 contains the Survey 1: National Health Authorities.

Additional file 2 contains the Survey 2: Manufacturers.

Additional file 3 contains the Survey 3: Poison Centers.

\section{References}

1. Chippaux J. Snakebites: appraisal of the global situation. Bull World Health Organ 1998; 76: 515-24.

2. Kasturiratne A, Wickremasinghe AR, de Silva N, Gunawardena NK, Pathmeswaran A, Premaratna R, et al. The global burden of snakebite: a literature analysis and modelling based on regional estimates of envenoming and deaths. PLoS Med 2008; 5: e218. doi: 10.1371/journal.pmed.0050218

3. World Health Organization (WHO). Rabies and Envenomings A neglected public health issue. Geneva: WHO; 2007. p. 1-38.

4. Harrison RA, Hargreaves A, Wagstaff SC, Faragher B, Lalloo DG. Snake envenoming: a disease of poverty. PLoS Negl Trop Dis 2009; 3: e569. doi: 10.1371/journal.pntd.0000569

5. Mohapatra B, Warrell DA, Suraweera W, Bhatia P, Dhingra $\mathrm{N}$, Jotkar RM, et al. Snakebite mortality in India: a nationally representative mortality survey. PLoS Negl Trop Dis 2011; 5: e1018. doi: 10.1371/journal.pntd.0001018

6. Gutiérrez JM, Theakston RDG, Warrell DA. Confronting the neglected problem of snake bite envenoming: the need for a global partnership. PLoS Med 2006; 3: e150. doi: 10.1371/ journal.pmed.0030150

7. Calvete JJ, Sanz L, Pla D, Lomonte B, Gutiérrez JM. Omics meets biology: application to the design and preclinical assessment of antivenoms. Toxins (Basel) 2014; 6: 3388-405. doi: $\quad 10.3390 /$ toxins 6123388
8. Theakston RD, Warrel DA. Crisis in snake antivenom supply for Africa. Lancet 2000; 356: 2104. doi: 10.1016/s01406736(05)74319-1

9. Chippaux J. Estimating the global burden of snakebite can help to improve management. PLoS Med 2008; 5: e221. doi: 10.1371/ journal.pmed.0050221

10. Gutiérrez JM, Williams D, Fan HW, Warrell DA. Snakebite envenoming from a global perspective: Towards an integrated approach. Toxicon 2010; 56: 1223-35. doi: 10.1016/j. toxicon.2009.11.020

11. Gutiérrez JM, Burnouf T, Harrison RA, Calvete JJ, Kuch U, Warrell DA. A multicomponent strategy to improve the availability of antivenom for treating snakebite envenoming. Bull World Health Organ 2014; 92: 526-32. doi: 10.2471/blt.13.132431

12. Williams DJ, Gutiérrez J-M, Calvete JJ, Wüster W, Ratanabanangkoon K, Paiva $\mathrm{O}$, et al. Ending the drought: new strategies for improving the flow of affordable, effective antivenoms in Asia and Africa. J Proteomics 2011; 74: 1735-67. doi: 10.1016/j.jprot.2011.05.027

13. Gutiérrez JM, Warrell DA, Williams DJ, Jensen S, Brown N, Calvete JJ, et al. The need for full integration of snakebite envenoming within a global strategy to combat the neglected tropical diseases: the way forward. PLoS Negl Trop Dis 2013; 7: e2162. doi: 10.1371/journal.pntd.0002162

14. Gutiérrez JM, León G, Burnouf T. Antivenoms for the treatment of snakebite envenomings: the road ahead. Biologicals 2011; 39: 129-42. doi: 10.1016/j.biologicals.2011.02.005

15. Morals V, Massaldi H. Snake Antivenoms: Adverse Reactions and Production Technology. J Venom Anim Toxins incl Trop Dis 2008; 15: 3. doi: 10.1590/s1678-91992009000100002

16. Bawaskar HS. Snake venoms and antivenoms: critical supply issues. J Assoc Physicians India 2004; 52: 11-3.

17. Brown NI. Consequences of neglect: analysis of the sub-Saharan African snake antivenom market and the global context. PLoS Negl Trop Dis 2012; 6: e1670. doi: 10.1371/journal.pntd.0001670

18. World Health Organization (WHO). Guidelines for the production control and regulation of snake antivenom immunoglobulins. Geneva: WHO; 2008.

19. World Health Organization (WHO). Venomous snakes distribution and species risk categories [Internet]. 2010. Available from: http://apps.who.int/bloodproducts/snakeantivenoms/database/

20. León G, Segura Á, Gómez A, Hernandez A, Navarro D, Villalta $\mathrm{M}$, et al. Industrial Production and Quality Control of Snake Antivenoms. In: Gopalakrishnakone P, Calvete JJ, editors. Toxinology. Dordrecht: Springer; 2021. p. 1-22.

21. Isbister GK. Snake antivenom research: the importance of case definition. Emerg Med J 2005; 22: 399-400. doi: 10.1136/ emj.2004.022251

22. Warrell DA. Snake bite. Lancet 2010; 375: 77-88. doi: 10.1016/ s0140-6736(09)61754-2 


\section{Appendix 1}

List of manufacturers contacted, as listed in the WHO database for antivenom producers

\begin{tabular}{|c|c|c|c|}
\hline No. & Country & Manufacturer name & Comments \\
\hline 1 & Algeria & Institut Pasteur d'Algerie & Invalid contact \\
\hline 2 & Argentina & Instituto Biologico Argentino "BIOL" & Invalid contact \\
\hline 3 & Argentina & Instituto Nacional de Produccion de Biologicos & No response \\
\hline 4 & Argentina & Laboratorio Central de Salud Publica & Invalid contact \\
\hline 5 & Australia & CSL Limited & Responded \\
\hline 6 & Bolivia & Instituto Nacional de Laboratorios de Salud & Invalid contact \\
\hline 7 & Brazil & Centro de Producao e Pesquisas de Immunobiol & Invalid contact \\
\hline 8 & Brazil & Fundacao Ezequiel Dias (FUNED) & Invalid contact \\
\hline 9 & Brazil & Instituto Butantan & No response \\
\hline 10 & Brazil & Instituto Vital Brazil S.A. & Invalid contact \\
\hline 11 & China & Lanzhou Institute of Biological Products & Responded \\
\hline 12 & China & Shanghai Institute Biological Technology Co., Ltd. & Invalid contact \\
\hline 13 & Colombia & Laboratorios PROBIOL S.A. & No response \\
\hline 14 & Costa Rica & Instituto Clodomiro Picado & Invalid contact \\
\hline 15 & Croatia & Immunoloski Zavod & Invalid contact \\
\hline 16 & Ecuador & Instituto Nacional de Higiene y Medicina Tropical "Leopoldo Izquieta Pérez" & Invalid contact \\
\hline 17 & Egypt & VACSERA & No response \\
\hline 18 & France & Sanofi-Pasteur & No response \\
\hline 19 & India & Bharat Serums \& Vaccines & No response \\
\hline 20 & India & Biological E Limited & Invalid contact \\
\hline 21 & India & Haffkine Biopharmaceutical Corporation Ltd & Invalid contact \\
\hline 22 & India & VINS Bioproducts Ltd & Responded \\
\hline 23 & Indonesia & BioFarma & No response \\
\hline 24 & Iran & Razi Vaccine \& Serum Research Institute & Invalid contact \\
\hline 25 & Japan & Japan Snake Institute & No response \\
\hline 26 & Japan & The Chemo-Sero-Therapeutic Research Institute (Kaketsuken) & Invalid contact \\
\hline 27 & Korea & Korea Vaccine Co. Ltd. & No response \\
\hline 28 & Mexico & Instituto Bioclon & No response \\
\hline 29 & Mexico & Instituto Nacional de Higiene (Birmex) & No response \\
\hline 30 & Myanmar & Myanmar Pharmaceutical Factory & No response \\
\hline 31 & Pakistan & National Institute of Health & Responded \\
\hline 32 & Peru & Instituto Nacional de Salud. Centro Nacional de Productos Biológicos & Invalid contact \\
\hline 33 & Philippines & Biologicals Manufacturing Division (Research Institute for Tropical Medicine) & Responded \\
\hline 34 & Poland & BIOMED (Wytwornia Surowic i Szczepionek) & No response \\
\hline 35 & Russia & Federal State Company for Immunobiological Medicines "Microgen" & Invalid contact \\
\hline 36 & Saudi Arabia & National Antivenom \& Vaccine Production Center (NAVPC) & No response \\
\hline 37 & Serbia & Institute of Virology, Vaccine and Sera TORLAK & No response \\
\hline 38 & South Africa & South African Vaccine Producers (SAVP) & No response \\
\hline 39 & Taiwan & National Institute of Preventive Medicine & Invalid contact \\
\hline 40 & Thailand & Queen Saovabha Memorial Institute & Responded \\
\hline 41 & Tunisia & Institut Pasteur de Tunis & No response \\
\hline 42 & Uruguay & Instituto de Higiene & Invalid contact \\
\hline 43 & USA & Protherics Inc & Invalid contact \\
\hline 44 & USA & Wyeth & Invalid contact \\
\hline 45 & Venezuela & Centro de Biotecnologia & Invalid contact \\
\hline 46 & Vietnam & Institute of Vaccines and Biological Substances (IVAC) & Invalid contact \\
\hline
\end{tabular}


Appendix 2

List of National Health Authorities (NHAs) contacted (country name only), based on attendance list of the WHO Bi-Regional Workshop on Production, Control, and Regulation of Antivenoms (2008, Jakarta)

\begin{tabular}{|c|c|c|}
\hline No. & Country & Comment \\
\hline 1 & Benin & No response \\
\hline 2 & Bangladesh & No response \\
\hline 3 & Brazil & No response \\
\hline 4 & Burkina Faso & Responded \\
\hline 5 & Cambodia & Responded \\
\hline 6 & China & No response \\
\hline 7 & Egypt & No response \\
\hline 8 & Ethiopia & No response \\
\hline 9 & Indonesia & No response \\
\hline 10 & Iran & No response \\
\hline 11 & Japan & Responded \\
\hline 12 & Kenya & No response \\
\hline 13 & Malaysia & Responded \\
\hline 14 & Nepal & Responded \\
\hline 15 & Niger & No response \\
\hline 16 & Nigeria & Responded \\
\hline 17 & Philippines & No response \\
\hline 18 & Saudi Arabia & No response \\
\hline 19 & Tanzania & No response \\
\hline 20 & Thailand & Responded \\
\hline 21 & Uganda & Responded \\
\hline 22 & Vietnam & Responded \\
\hline 23 & Zimbabwe & Responded \\
\hline
\end{tabular}

